\title{
Diabetes Melito Tipo 1 no Contexto das Poliendocrinopatias Auto-imunes
}

revisão

\section{MÁrcia S. QueIRoz}

Grupo de Diabetes do Serviço de Endocrinologia e Laboratório de Investigação Médica - 25 (LIM-25) do Hospital das Clínicas, Faculdade de Medicina da Universidade São Paulo, SP, Brasil.
Recebido em 14/01/2008 Aceito em 18/01/2008

\author{
RESUMO
}

Três entidades clínicas distintas, causadas por desarranjos genéticos, estão associadas a múltiplas desordens auto-imunes: síndrome linfoproliferativa auto-imune, poliendocrinopatias auto-imunes (APSs) e desregulação imune, poliendocrinopatia, enteropatia ligada ao $X$ (IPEX). $O$ diabetes melito autoimune ou tipo 1 (DM1) pode estar presente nas APSs e na IPEX. A APS-1 caracteriza-se pela associação de candidíase crônica, hipoparatireoidismo e insuficiência adrenal auto-imune ou idiopática (doença de Addison), no entanto, o diabetes melito tipo 1 pode estar presente em até $12 \%$ dos casos. A APS-2 inclui a doença de Addison (sempre presente), a doença tireoidiana auto-imune e o diabetes melito tipo 1, enquanto na APS-3 a doença tireoidiana se associa a outra doença imunológica, excluindo a insuficiência adrenal e o hipoparatireoidismo, e a APS-4 é composta por combinações diferentes das descritas anteriormente. Já a IPEX caracteriza-se por alteração rara da regulação auto-imune que resulta doenças auto-imunes de início precoce, envolvendo pâncreas, tireóide, intestino com diarréia secretora grave, eczema e anemia. O diagnóstico e o tratamento das doenças componentes das poliendocrinopatias e da IPEX são semelhantes ao da patologia na forma isolada. (Arq Bras Endocrinol Metab 2008;52/2:198-204)

Descritores: Diabetes melito tipo 1; Poliendocrinopatias

\section{ABSTRACT}

\section{Type 1 Diabetes and Autoimmune Polyendocrine Syndromes.}

Type 1 diabetes (T1D) is associated with autoimmune thyroid disease (AIT), celiac disease (CD), Addison's disease (AD), and other autoimmune diseases. These diseases can occur simultaneously in defined syndromes with distinct pathophysiology and characteristics: autoimmune polyendocrine syndromes (APSs) and the immunodysregulation polyendocrinopathy enteropathy $X$-linked syndrome (IPEX). APSs were initially defined as a multiple endocrine gland insufficiency associated to an autoimmune disease in a patient. APS- 1 is characterized by the evidence of chronic candidiasis, chronic hypoparathyroidism, $A D$ and T1D could be present as part of this syndrome. The combination of autoimmune adrenal insufficiency with AIT and/or type 1 autoimmune diabetes mellitus defines APS-2. AIT associated to other autoimmune diseases (excluding AD and/ or hypoparathyroidism) are the main characteristics of APS-3. Different clinical combinations of autoimmune diseases which were not included in the previous groups are the characteristics of APS-4. IPEX is a recessive disorder characterized by the neonatal onset of T1D, infections, enteropathy, thrombocytopenia and anemia, as well as endocrinopathy, eczema and cachexia. These disorders are not common, but their consequences can be life threatening when the diagnosis is overlooked, and the treatment is the same prescribed for isolated disease presentation. (Arq Bras Endocrinol Metab 2008;52/2:198-204)

Keywords: Type 1 diabetes mellitus; Autoimmune polyendocrine syndromes 


\section{INTRODUÇÃO}

$\mathrm{T}$ RÊS ENTIDADES CLÍNICAS DISTINTAS, causadas por desarranjos genéticos, estão associadas a múltiplas desordens auto-imunes: 1) síndrome linfoproliferativa auto-imune; 2) poliendocrinopatias auto-imunes (autoimmune polyglandular syndrome [APS]); e 3) desregulação imune, poliendocrinopatia, enteropatia ligada ao X (immune dysregulation, polyendocriopathy, enteropathy, X-linked ou IPEX) (1). O diabetes melito autoimune ou tipo 1 (DMl) pode estar presente na APS e na $\operatorname{IPEX}$ (Tabela 1).
As poliendocrinopatias são caracterizadas pela associação de duas ou mais desordens auto-imunes. Em 1980, Neufeld e Blizzard organizaram e classificaram as poliendocrinopatias em quatro grupos diferentes ( $\mathrm{Ta}$ bela 2) $(3,4)$. A APS-1 caracteriza-se pela associação de candidíase crônica, hipoparatireoidismo e insuficiência adrenal auto-imune ou idiopática (doença de Addison); a APS-2 inclui a doença de Addison (sempre presente), a doença tireoidiana auto-imune e o DMl; enquanto na APS-3 a doença tireoidiana se associa a outra doença imunológica, excluindo a insuficiência adrenal e o hipoparatireoidismo (Figura 1); e a APS-4 é composta por

Tabela 1. Características das síndromes auto-imunes poliendocrinas.

\begin{tabular}{|c|c|c|c|}
\hline & APS-1 & APS-2 & IPEX \\
\hline Prevalência & Rara & Comum & Muito rara \\
\hline Início & Infância & Infância até idade adulta & Período neonatal \\
\hline Genética & AIRE (cromossomo 21) & Poligênica & FOXP3 \\
\hline Padrão de herança & Recessivo & & Ligado ao X \\
\hline HLA & $\begin{array}{l}\text { Diabetes - risco } \\
\text { aumentado com HLA-DQ6 }\end{array}$ & $\begin{array}{l}\text { HLA-DQ2 e DQ8 } \\
\text { HLA-DRB } 1^{*} 0404\end{array}$ & Sem associação \\
\hline Imunodeficiência & $\begin{array}{l}\text { Anesplenismo, suscetibilidade } \\
\text { à candidíase }\end{array}$ & Nenhuma & $\begin{array}{l}\text { Perda de células T } \\
\text { regulatórias }\end{array}$ \\
\hline Associação com DM & $\operatorname{Sim}(18 \%)$ & $\operatorname{Sim}(20 \%)$ & Sim (maioria) \\
\hline Fenótipo comum & $\begin{array}{l}\text { Candidíase, hipoparatireoidismo, } \\
\text { doença de Addison }\end{array}$ & $\begin{array}{l}\text { Doença de Addison, } \\
\text { DM1, tireoidite }\end{array}$ & $\begin{array}{l}\text { Diabetes neonatal, } \\
\text { malabsorção }\end{array}$ \\
\hline
\end{tabular}

Modificado de Eisenbarth e Gottlieb, 2004 (2).

Tabela 2. Classificação das poliendocrinopatias auto-imunes.

\begin{tabular}{ll}
\hline Classificação & Patologias associadas \\
\hline APS tipo 1 & Candidíase crônica \\
& Hipoparatireoidismo \\
& Insuficiência adrenal auto-imune \\
& Insuficiência adrenal auto-imune (deve estar sempre presente) \\
\hline APS tipo 2 & Doença tireoidiana auto-imune \\
\hline APS tipo 3 & Diabetes melito tipo 1 \\
& Doença tireoidiana auto-imune \\
& Excluindo: insuficiência adrenal, hipoparatireoidismo e candidíase crônica \\
\hline APS tipo 4 & Associação de 2 ou mais doenças auto-imunes, com padrão diferente da APS-1, 2 ou 3 \\
\hline
\end{tabular}

Modificado de Neufeld e cols., 1980 (3) 


\begin{tabular}{|c|c|c|c|}
\hline \multicolumn{4}{|c|}{ Poliendocrinopatia auto-imune tipo 3 (APS-3) } \\
\hline \multicolumn{4}{|c|}{ Doenças tireoidianas auto-imunes } \\
\hline $\begin{array}{l}\text { Tireoidite de Hashimoto } \\
\text { Mixedema idiopático } \\
\text { Tireoidite assintomática }\end{array}$ & Exoftalmopatia endócrina & Doença de Graves & \\
\hline+ & + & + & + \\
\hline $\begin{array}{l}\text { Diabetes melito tipo } 1 \\
\text { Síndrome de Hirata } \\
\text { Falência ovariana } \\
\text { prematura } \\
\text { Hipofisite linfocítica } \\
\text { Neuro-hipofisite }\end{array}$ & $\begin{array}{l}\text { Gastrite atrófica } \\
\text { Anemia perniciosa } \\
\text { Doença celíaca } \\
\text { Doença inflamatória intestinal } \\
\text { Hepatite auto-imune } \\
\text { Cirrose biliar primária } \\
\text { Colangite esclerosante }\end{array}$ & $\begin{array}{l}\text { Vitiligo } \\
\text { Alopecia } \\
\text { Trombocitopenia auto-imune } \\
\text { Anemia hemolítica auto-imune } \\
\text { Síndrome antifosfolípides } \\
\text { Miastenia gravis } \\
\text { Síndrome Stiff-man } \\
\text { Esclerose múltipla }\end{array}$ & $\begin{array}{l}\text { Lúpus eritematoso } \\
\text { sistêmico } \\
\text { Lúpus eritematoso } \\
\text { difuso } \\
\text { Doenças mistas do } \\
\text { tecido conectivo } \\
\text { Artrite reumatóide } \\
\text { Artrite reacional } \\
\text { Síndrome de } \\
\text { Sjögren } \\
\text { Vasculites }\end{array}$ \\
\hline APS-3 A & APS-3 B & APS-3 C & APS-3 D \\
\hline
\end{tabular}

Figura 1. Nova classificação da APS-3 e subgrupos proposta por Betterle e cols., 2002 (5).

combinações diferentes das descritas anteriormente. No entanto, com o surgimento e o aprimoramento de técnicas para dosagem de anticorpos, Betterle e cols. propuseram uma nova classificação para APS-2, com sua divisão em subclínica e potencial. Na APS-2 subclínica observa-se a manifestação clínica de uma doença auto-imune associada a um ou mais marcadores sorológicos das outras patologias que integram a síndrome, porém sem expressão clínica evidente de dano ao órgão-alvo, por exemplo, pacientes com insuficiência adrenal, anticorpos antiperoxidase ou antitireoglobulina e doença tireoidiana subclínica ou pacientes com insuficiência adrenal, anticorpos contra ilhotas e intolerância à glicose. Enquanto na APS-2 potencial a doença auto-imune clinicamente deflagrada estaria associada a anticorpos específicos das doenças que compõem a tríade, sem alteração de função do órgão-alvo (Tabela 3). Outras patologias auto-imunes podem estar presentes na APS-2, conforme mostrado na Tabela 4.
Tabela 3. Combinações possiveis para APS-2 potencial.

\begin{tabular}{|c|c|}
\hline Doenças crônicas & Sorologia \\
\hline Doença de Addison & $\begin{array}{l}\text { Anticorpos tireoidianos } \\
\text { Ac. anti-ICA } \\
\text { Ac. anti-GAD }\end{array}$ \\
\hline Doença tireoidiana auto-imune & $\begin{array}{l}\text { Ac. anti-CA } \\
\text { Ac. anti-21-OH }\end{array}$ \\
\hline Diabetes melito tipo 1 & $\begin{array}{l}\text { Ac. anti-CA } \\
\text { Ac. anti-21-OH }\end{array}$ \\
\hline $\begin{array}{l}\text { Doença tireoidiana auto-imune } \\
\text { e diabetes melito tipo } 1\end{array}$ & $\begin{array}{l}\text { Ac. anti-CA } \\
\text { Ac. anti-21-OH }\end{array}$ \\
\hline Nenhuma & $\begin{array}{l}\text { Ac. anti-CA } \\
\text { Ac. anti-21-OH } \\
\text { Anticorpos tireoidianos } \\
\text { Ac. anti-ICA } \\
\text { Ac. anti-GAD }\end{array}$ \\
\hline
\end{tabular}

Ac. anti-ICA: anticorpos antilihota; anti-GAD: anticorpos antiácido

decarboxilase glutâmico; anti-21-OH: anticorpos anti-21-hidroxilase; anti-CA anticorpos anticórtex adrenal. Modificado de Betterle \& Zanchetta, 2003 (3) 
Tabela 4. Patologias menores associadas à poliendocrinopatia auto-imune tipo-2*.

\begin{tabular}{lc}
\hline Condições & Pacientes acometidos \\
\hline Vitiligo & $4,5 \%$ a $11 \%$ \\
\hline Atrofia gástrica crônica, associada ou não à anemia perniciosa & $4,5 \%$ a $11 \%$ \\
\hline Hipogonadismo hipergonadotrófico & $4 \%$ a $9 \%$ \\
Hepatite auto-imune & $4 \%$ \\
\hline Alopecia & $1 \%$ a $4 \%$ \\
Hipofisite & $<1 \%$ \\
Miastenia gravis & $<1 \%$ \\
Artrite reumatóide & $<1 \%$ \\
\hline Síndrome de Sjögren & $<1 \%$ \\
\hline Púrpura trombocitopênica & $<1 \%$ \\
\hline
\end{tabular}

* Modificado de Betterle e cols., 2004 (6).

A IPEX foi inicialmente descrita por Powell e col. (7) e caracteriza-se por alteração rara da regulação auto-imune que resulta doenças auto-imunes de início precoce, envolvendo pâncreas, tireóide, intestino com diarréia secretora severa, eczema e anemia. $\mathrm{O}$ acometimento das paratireóides e adrenais é raro nesta síndrome (8).

\section{EPIDEMIOLOGIA}

A APS-1 é uma síndrome rara, porém mais freqüente em algumas comunidades e regiões geográficas, sua prevalência varia de 1:9.000 a 1:200.000 habitantes, a proporção de mulheres para homens acometidos varia de 0,8 a $2,4(3)$.

A síndrome poliglandular auto-imune tipo 2 (APS-2) também é uma condição clínica rara com prevalência estimada de 1,4 a 2,0 casos por 100.000 habitantes (5), ocorre entre 30 e 40 anos de idade e afeta 2 a 3,7 vezes mais mulheres do que homens $(9,10)$.

Os aspectos epidemiológicos da APS-3 e da APS-4 não estão bem definidos. Betterle e cols. revisaram 263 pacientes com doença de Addison e encontraram 13 casos $(5 \%)$ que puderam ser diagnosticados como APS-4, nesta casuística a idade média foi de 36 anos e com predomínio de mulheres (5).

A IPEX é extremamente rara, com transmissão genética ligada ao cromossomo $\mathrm{X}$ e acomete basicamente indivíduos do sexo masculino (11).

\section{GENÉTICA}

A síndrome poliglandular auto-imune apresenta padrão de herança genética complexo (12). A APS-1, tanto na forma familiar como na esporádica, tem caráter autossômico recessivo e associa-se a vários alelos do sistema HLA (buman leucocyte antigen) classes I e II, principalmente o HLA-A28, HLA-A3 (em pacientes com APS-1 e falência ovariana e o HLA-DR5) $(3,13,14)$.

A herança da APS-2 é autossômica dominante com penetrância incompleta, com forte ligação aos haplótipos HLA classe II DR3 (DQB*0201) e DR4 $\left(\mathrm{DQB1}{ }^{*} 0302\right)(5,15)$. Outros genes, como o polimorfismo do antígeno citotóxico 4 de linfócito T (CTLA-4), conferem suscetibilidade à doença de Addison autoimune relacionada à APS-2, mas não na APS-1 (16), e as mutações no gene AIRE, que são tipicamente encontradas na APS-1, não foram descritas em pacientes com insuficiência adrenal e APS-2 (17).

Até o momento não se conhece o padrão genético nas APS-3 e 4 (3).

Sabe-se que a IPEX é causada por mutações no gene FOXP3 locado braço curto do cromossomo X, Xp11.23 (18), responsável pela codificação da proteína scurfin, necessária para o desenvolvimento das células T regulatórias CD4+ CD25+, na sua ausência as células CD4+ ativadas não são suprimidas pelas células reguladoras (CD4 FOXP3+), permitindo a proliferação de linfócitos ativados responsivos contra os próprios antígenos com conseqüente destruição tecidual $(19,20)$. 


\section{DIABETES MELITO NO CONTEXTO DAS POLIENDOCRINOPATIAS}

\section{APS-1}

Apesar da alta prevalência de auto-anticorpos, principalmente contra ilhotas pancreáticas (anti-ICA, 18\% a $30 \%$ ) e antidescarboxilase glutâmica (anti-GAD, 41\%), o DM está presente em $0 \%$ a $12 \%$ dos casos de APS- 1 (21), essa discrepância talvez possa ser explicada pela falta de predisposição genética ou por auto-anticorpos reconhecerem epítopos diferentes dos anticorpos presentes no DMl clássico (22).

\section{APS-2}

Na APS-2, o DMl é considerado um dos componentes da síndrome, em que a insuficiência adrenal auto-imune (IAA) pode estar associada à doença tireoidiana autoimune (mais freqüente) e ao DMl (Tabela 5). Em uma série de 146 pacientes com insuficiência adrenal isolada ou como parte de uma poliendocrinopatia, Betterle e cols. (6) relataram a presença de DM em $23 \%$ dos casos, nas seguintes associações: a) 10,9\% dos pacientes tinham IAA e DMl; b) 9,6\% dos pacientes tinham IAA, tireoidite crônica e $\mathrm{DMl}$; e c) $2 \%$ dos pacientes tinham DM, $\mathrm{AD}$ e doença de Graves. As doenças que compõem a APS-2 tendem a se desenvolver em uma seqüência específica, com o DMl diagnosticado geralmente antes da insuficiência adrenal, enquanto a doença tireoidiana pode desenvolver-se antes, ao mesmo tempo ou logo após a insuficiência adrenal (9).

\section{APS-3}

A associação DMl e doença tireoidiana auto-imune é a forma mais freqüente de apresentação da APS-3 (3A). A evolução do diabetes pode ser extremamente variável, desde lentamente progressiva e de caráter estável até a perda completa da secreção de insulina logo após o diagnóstico $(23,24)$. Estudos epidemiológicos mostram que $20 \%$ dos pacientes $\mathrm{DMl}$ desenvolvem doença tireoidiana auto-imune (25).

\section{IPEX}

O DMI é a patologia endocrinológica mais comum na IPEX, freqüentemente diagnosticado no primeiro ano de vida, em alguns casos a intolerância à glicose já está presente ao nascimento. Os pacientes diabéticos geralmente têm anticorpos antiilhota, bem como inflamação intersticial crônica e infiltrado linfocitário no pâncreas, com completa destruição das ilhotas (26).

\section{APRESENTAÇÃo CLÍNICA}

Os sinais e sintomas da poliendocrinopatias múltiplas são semelhantes aos da doença isolada e o padrão de associação das diversas patologias caracterizará a síndrome, conforme descrito na Tabela 2 . Neste contexto, o DM pode ser a primeira patologia auto-imune diagnosticada por meio dos sintomas clássicos de poliúria, polidipsia, polifagia, emagrecimento, evoluindo com descompensação e cetoacidose ou pode ser parte das

Tabela 5. Freqüências das patologias principais na poliendocrinopatia auto-imune-2.

\begin{tabular}{lcccc}
\hline $\begin{array}{l}\text { Autor } \\
\text { (ano) }\end{array}$ & $\begin{array}{c}\text { Neufeld e cols. } \\
1980 \text { (4) }\end{array}$ & $\begin{array}{c}\text { Papadopoulos \& } \\
\text { Hallengren 1990 (27) }\end{array}$ & $\begin{array}{c}\text { Betterle e cols. } \\
\text { 2002 (5) }\end{array}$ & $\begin{array}{c}\text { Total } \\
\text { Número de pacientes }\end{array}$ \\
\hline Relação M/H & 224 & 22 & 107 & 351 \\
\hline Fãmiliares acometidos & Não avaliado & Não avaliado & 3,7 & 0 \\
\hline Doenças principais & $\%$ & $\%, 7$ & 0 \\
\hline IAA & 100 & 100 & $\%$ & 100 \\
\hline DTA & 69 & 73 & 100 & 62 \\
\hline DM1 & 52 & 41 & 30 & $30-52$ \\
\hline
\end{tabular}

IAA: insuficiência adrenal auto-imune; DTA: doença tireoidiana auto-imune; DM1: diabetes melito tipo 1. 
manifestações secundárias, geralmente de caráter mais insidioso e detectado por alteração no teste oral de tolerância à glicose.

\section{DIAGNÓSTICO}

Quando o DMl integra a poliendocrinopatia autoimune, os critérios para diagnóstico são os mesmos delineados nos diversos consensos, ou seja, valor da glicemia de jejum acima de $126 \mathrm{mg} / \mathrm{dL}$ ou glicemia 2 horas após sobrecarga oral com $75 \mathrm{~g}$ de glicose anidra maior ou igual a $200 \mathrm{mg} / \mathrm{dL}$ e presença de anticorpos antiilhota (anti-ICA), antiácido decarboxilase glutâmico (anti-GAD), antiinsulina e antiproteína tirosina-fosfatase (anti-IA2)(28). Os auto-anticorpos desenvolvem-se seqüencialmente e os anticorpos antiinsulina são os primeiros a serem expressos, principalmente em crianças pequenas $(29,30)$.

Na IPEX, o diagnóstico deve ser considerado em pacientes jovens, do sexo masculino com: diarréia intratável, atrofia vilositária, déficit de crescimento, dermatite, hipotireoidismo e diabetes de início precoce (1).

\section{TRATAMENTO}

A terapia de reposição hormonal para as doenças componentes das poliendocrinopatias é semelhante à terapia da doença na sua forma isolada, no entanto, em pacientes diagnosticados portadores da APS-2 ou com diabetes e hipotireoidismo auto-imune, a avaliação da função adrenal é essencial antes de se iniciar a reposição com hormônio tireoidiano, pois a tiroxina aumenta o metabolismo hepático de corticosteróides, o que pode desencadear uma crise adrenal e colocar o paciente em situação de risco (31).

O tratamento do DM na APS, como na IPEX, segue os mesmos preceitos de insulinização adotados atualmente, ou seja, insulinização plena por meio da terapia basal - bolus, em que $50 \%$ da dose total diária de insulina corresponderão à insulina basal e 50\% serão administrados na forma de bólus pré-prandial.

Na IPEX, o prognóstico é ruim, por causa da gravidade das patologias envolvidas. $\mathrm{O}$ tratamento sintomático inclui nutrição parenteral e, se necessário, transfusão de concentrado de hemácias e plaquetas, além da insulinização plena. Atualmente, somente o transplante de medula óssea é efetivo na cura de pacien- tes com IPEX, mas na maioria dos casos as endocrinopatias persistirão, especialmente o DM, em virtude da destruição maciça das células-beta produtoras de insulina $(1,32-34)$.

\section{CONCLUSÃO}

As poliendocrinopatias têm largo espectro de apresentação, o DMl pode ser a manifestação inicial, uma vez que outras doenças auto-imunes podem estar em fase latente, a necessidade de investigação adicional com a dosagem de auto-anticorpos permanece controversa. Atualmente, a Sociedade Brasileira de Diabetes (35) recomenda a investigação para doença celíaca no diagnóstico do DMl ou na presença de sintomas e a dosagem anual de TSH para diagnóstico de doença tireoidiana, não sendo recomendada a pesquisa sistemática para doença adrenal ou de outras patologias componentes das poliendocrinopatias auto-imunes.

\section{REFERÊNCIAS}

1. Torgerson TR, Ochs HD. Immune dysregulation, polyendocrinopathy, enteropathy, X-linked: forkhead box protein $3 \mathrm{mu}$ tations and lack of regulatory T cells. J Allergy Clin Immunol. 2007;120(4):744-50.

2. Eisenbarth GS, Gottlieb PA. Autoimmune polyendocrine syndromes. N Engl J Med. 2004;350(20):2068-79.

3. Betterle C, Zanchetta R. Update on autoimmune polyendocrine syndromes (APS). Acta Biomed. 2003;74(1):9-33.

4. Neufeld M, Maclaren N, Blizzard R. Autoimmune polyglandular syndromes. Pediatr Ann. 1980;9(4):154-62.

5. Betterle C, Dal Pra C, Mantero F, Zanchetta R. Autoimmune adrenal insufficiency and autoimmune polyendocrine syndromes: autoantibodies, autoantigens, and their applicability in diagnosis and disease prediction. Endocr Rev. 2002;23(3):327-64.

6. Betterle C, Greggio NA, Volpato M. Clinical review 93: autoimmune polyglandular syndrome type 1. J Clin Endocrinol Metab. 1998;83(4):1049-55.

7. Powell BR, Buist NR, Stenzel P. An X-linked syndrome of diarrhea, polyendocrinopathy, and fatal infection in infancy. J Pediatr. 1982;100(5):731-7.

8. Wildin RS, Smyk-Pearson S, Filipovich AH. Clinical and molecular features of the immunodysregulation, polyendocrinopathy, enteropathy, $X$ linked (IPEX) syndrome. J Med Genet. 2002;39(8):537-45.

9. Schatz DA, Winter WE. Autoimmune polyglandular syndrome. II: clinical syndrome and treatment. Endocrinol Metab Clin North Am. 2002;31(2):339-52.

10. Betterle C, Volpato M, Greggio AN, Presotto F. Type 2 polyglandular autoimmune disease (Schmidt's syndrome). J Pediatr Endocrinol Metab. 1996;9 Suppl 1:113-23.

11. Wildin RS, Freitas A. IPEX and FOXP3: clinical and research perspectives. J Autoimmun. 2005;25 Suppl:56-62. 
12. Vyse TJ, Todd JA. Genetic analysis of autoimmune disease. Cell. 1996;85(3):311-8.

13. Ahonen P. Autoimmune polyendocrinopathy-candidosis-ectodermal dystrophy (APECED): autosomal recessive inheritance. Clin Genet. 1985;27(6):535-42.

14. Ahonen P, Koskimies S, Lokki ML, Tiilikainen A, Perheentupa J. The expression of autoimmune polyglandular disease type I appears associated with several HLA-A antigens but not with HLA-DR. J Clin Endocrinol Metab. 1988;66(6):1152-7.

15. Robles DT, Fain PR, Gottlieb PA, Eisenbarth GS. The genetics of autoimmune polyendocrine syndrome type II. Endocrinol Metab Clin North Am. 2002;31(2):353-vii.

16. Kemp EH, Ajjan RA, Husebye ES, Peterson P, Uibo R, Imrie $H$, et al. A cytotoxic T lymphocyte antigen-4 (CTLA-4) gene polymorphism is associated with autoimmune Addison's disease in English patients. Clin Endocrinol (Oxf). 1998;49(5):609-13.

17. Halonen M, Eskelin P, Myhre AG, Perheentupa J, Husebye ES, Kampe O, et al. AIRE mutations and human leukocyte antigen genotypes as determinants of the autoimmune polyendocrinopathy-candidiasis-ectodermal dystrophy phenotype. J Clin Endocrinol Metab. 2002;87(6):2568-74.

18. Bennett CL, Christie J, Ramsdell F, Brunkow ME, Ferguson PJ, Whitesell $L$, et al. The immune dysregulation, polyendocrinopathy, enteropathy, X-linked syndrome (IPEX) is caused by mutations of FOXP3. Nat Genet. 2001;27(1):20-1.

19. Fontenot JD, Rudensky AY. A well adapted regulatory contrivance: regulatory $T$ cell development and the forkhead family transcription factor Foxp3. Nat Immunol. 2005;6(4):331-7.

20. Yagi H, Nomura T, Nakamura K, Yamazaki S, Kitawaki T, Hori S, et al. Crucial role of FOXP3 in the development and function of human CD25+CD4+ regulatory T cells. Int Immunol 2004; 16(11):1643-56.

21. Perheentupa J, Miettinen A. Type 1 autoimmune polyglandular disease. Ann Med Interne (Paris). 1999;150(4):313-25.

22. Betterle C, Lazzarotto F, Presotto F. Autoimmune polyglandular syndrome type 2: the tip of an iceberg? Clin Exp Immunol. 2004;137(2):225-33.

23. Oki K, Yamane K, Koide J, Mandai K, Nakanishi S, Fujikawa R, et al. A case of polyglandular autoimmune syndrome type III complicated with autoimmune hepatitis. Endocr J. 2006;531 5):705-9.

24. Shimomura $H$, Nakase $Y$, Furuta $H$, Nishi M, Nakao T, Hanabusa $T$, et al. A rare case of autoimmune polyglandular syndrome type 3. Diabetes Res Clin Pract. 2003;61(2):103-8.
25. Kordonouri O, Klinghammer A, Lang EB, Gruters-Kieslich A, Grabert M, Holl RW. Thyroid autoimmunity in children and adolescents with type 1 diabetes: a multicenter survey. Diabetes Care. 2002;25(8):1346-50.

26. Levy-Lahad E, Wildin RS. Neonatal diabetes mellitus, enteropathy, thrombocytopenia, and endocrinopathy: further evidence for an X-linked lethal syndrome. J Pediatr. 2001;138 (4):577-80.

27. Papadopoulos $\mathrm{KI}$, Hallengren $\mathrm{B}$. Polyglandular autoimmune syndrome type II in patients with idiopathic Addison's disease. Acta Endocrinol (Copenh). 1990;122(4):472-8.

28. Diagnosis and classification of diabetes mellitus. Diabetes Care. 2004;27 Suppl 1:S5-S10.

29. Barker JM, Goehrig SH, Barriga K, Hoffman M, Slover $R$, Eisenbarth GS, et al. Clinical characteristics of children diagnosed with type 1 diabetes through intensive screening and follow-up. Diabetes Care. 2004;27(6):1399-404.

30. Yu L, Rewers M, Gianani R, Kawasaki E, Zhang Y, Verge C, et al. Antiislet autoantibodies usually develop sequentially rather than simultaneously. J Clin Endocrinol Metab. 1996;81(12):4264-7.

31. Majeroni BA, Patel P. Autoimmune polyglandular syndrome, type II. Am Fam Physician. 2007;75(5):667-70.

32. Lucas KG, Ungar D, Comito M, Bayerl M, Groh B. Submyeloablative cord blood transplantation corrects clinical defects seen in IPEX syndrome. Bone Marrow Transplant. 2007;39 (1):55-6.

33. Mazzolari E, Forino C, Fontana M, D'Ippolito C, Lanfranchi A, Gambineri $E$, et al. A new case of IPEX receiving bone marrow transplantation. Bone Marrow Transplant. 2005;35(10):1033-4.

34. Rao A, Kamani N, Filipovich A, Lee SM, Davies SM, Dalal J, et al. Successful bone marrow transplantation for IPEX syndrome after reduced-intensity conditioning. Blood. 2007;109 (1):383-5.

35. Sociedade Brasileira de Diabetes. Atualização brasileira sobre diabetes. Rio de Janeiro: Diagraphic, 2005.

\section{Endereço para correspondência:}

Márcia S. Queiroz

Serviço de Endocrinologia - Depto. de Clínica Médica

Av. Dr. Éneas de Carvalho Aguiar, 255, $8^{\circ}$ andar

05403-000 - Cerqueira César, São Paulo, SP

E-mail: marciasqz@gmail.com 